\title{
Estado de los programas de trasplante renal en América Central y el Caribe
}

\author{
César Cuero ${ }^{1,2 *}$, Manuel Cerdas ${ }^{1,3}$, José J. Castillos ${ }^{1,4}$, Antonio Enamorado ${ }^{1,5}$, Carlos Henríquez ${ }^{1,6}$, \\ Agualuz Hernández ${ }^{1,7}$, Guillermo Álvarez ${ }^{1,8}$, Francisco González ${ }^{1,9}$ y Luis Morales ${ }^{1,10}$ \\ ${ }^{1}$ Grupo Coordinador de trasplante Renal de América Central y el Caribe, Asociación Centroamericana y del Caribe de Nefrología (ACECAN); \\ ${ }^{2}$ Organización Panameña de Trasplantes, Panamá; ${ }^{3}$ Hospital México, Caja Costarricense de Seguridad Social, Costa Rica; ${ }^{4}$ Hospital General de La \\ Plaza de la Salud, República Dominicana; ${ }^{5}$ Coordinación Nacional de Trasplantes, Cuba; ${ }^{6}$ Hospital Benjamín Bloom, El Salvador; ${ }^{7} \mathrm{Hospital} \mathrm{General}$ \\ de Enfermedades, Instituto Guatemalteco de Seguridad Social, Guatemala; ${ }^{8}$ Centro de Diagnóstico Medicina Avanzada y Telemedicina (CEDIMAT), \\ República Dominicana; ${ }^{9}$ Facultad de Medicina, Universidad de la República, Montevideo, Uruguay; ${ }^{10}$ Instituto Nacional de Ciencias Médicas y \\ Nutrición Manuel Zubirán, Ciudad de México, México
}

\section{Resumen}

Introducción: En agosto del 2017, se realizó la II Reunión de Centroamérica y del Caribe de Trasplante Renal, en El Salvador, que contó con los auspicios y la participación de la Sociedad de Trasplantes de América Latina y el Caribe (STALYC) y la Sociedad Latinoamericana de Nefrología e Hipertensión (SLANH) y en la que se analizó la situación de los trasplantes renales en la región, se llegaron a acuerdos, se sentaron las bases, entre otras cosas, de un registro de trasplante renal para toda Centroamérica y los países del Caribe hispano. Material y método: Se elaboró, con la participación de todos los países de la región y la colaboración de la STALYC y la SLANH, una encuesta, la cual, una vez consensuada, se envió a cada país que la llenó y la envió al grupo coordinador, que conjuntó los datos que incluyen los trasplantes hechos entre el 2012 y 2016, y fueron presentados a la reunión para su análisis. Resultados: Todos los países hacen trasplante renal. Todos hacen trasplante renal de donante vivo y algunos de fallecido. Todos cuentan con una ley de trasplantes, pero pocos con una estructura nacional de donación y trasplante y, por ende, con una lista de espera nacional. Solo dos países tienen un registro nacional de trasplante renal organizado y funcional. Se han hecho 11,443 trasplantes de donante vivo, 432 trasplantes de donante vivo privados y 3,254 trasplantes de donante fallecido. Conclusión: Se presenta la situación actual de los trasplantes renales en los países de América Central, República Dominicana y Cuba, sus dificultades, obstáculos y avances, así como los datos generales de los trasplantes renales hechos entre el 2012 y el 2016.

Palabras claves: Trasplante renal. Registro de trasplante. Inmunosupresión. Inducción.

\section{State of the kidney transplant program in Central America and the Caribbean}

\section{Abstract}

Introduction: In August 2017, the Second Meeting of Central America and the Caribbean of Renal Transplantation was held in El Salvador, with the sponsorship and participation of STALYC and LA SLANH, in which the situation was analyzed in greater depth of kidney transplants in the region, agreements were reached, the bases were laid, among other things, of a

\section{Correspondencia:}

${ }^{*}$ César Cuero

E-mail: ccuero@cwpanama.net licencia CC BY-NC-ND (http://creativecommons.org/licenses/by-nc-nd/4.0/)
Fecha de recepción: 02-04-2018

Fecha de aceptación: 27-06-2020

DOI: 10.24875/NEFRO.18000039
Disponible en internet: 17-12-2020

Nefro Latinoam. 2020;17:86-94 www.nefrologialatinoamericana.com 
kidney transplant registry for all of Central America and the countries of the Spanish Caribbean. Material and method: It was developed with the participation of all the countries of the region and the collaboration of Sociedad de Trasplantes de América Latina y el Caribe (STALYC) and Sociedad Latinoamericana de Nefrología e Hipertensión (SLANH) a survey, which once agreed, was sent to each country that filled it and sent it to the coordinating group, which He put together the data that includes the transplants made between 2012 and 2016, which were presented to the meeting for analysis. Results: All countries perform kidney transplants. Everyone does a kidney transplant from a living donor and some from a deceased. All have a transplant law, but few have a national donation and transplant structure and therefore a national waiting list. Only two countries have a national registry of organized and functional kidney transplants. 11,443 live donor transplants, 432 private live donor transplants and 3,254 deceased donor transplants. Conclusion: The current situation of renal transplants in the countries of Central America, the Dominican Republic and Cuba is presented; it is difficulties, obstacles, advances and the general data of kidney transplants done from 2012 to 2016.

Key words: Kidney transplant. Registration of transplant. Immunosuppression. Induction.

\section{Introducción}

La enfermedad renal crónica (ERC) constituye un gran problema de salud que afecta cerca del $10 \%$ de la población mundial ${ }^{1,2}$. Su crecimiento sostenido preocupa a las instituciones de salud tanto a nivel mundial como de nuestra región, así como a las sociedades nefrológicas multinacionales, que la definen como una verdadera epidemia de índole global ${ }^{2,3}$, que muestra una prevalencia que varía según las regiones geográficas $^{1,3}$. América Latina no escapa a esta realidad, con el agravante de la limitación económica, que conlleva la limitación del acceso a la atención y al tratamiento de los pacientes con ERC ${ }^{4}$.

Numerosos estudios han demostrado que el trasplante renal mejora la sobrevida a largo plazo de los pacientes con ERC, al compararlo con la diálisis ${ }^{5-7}$. Es la mejor opción de tratamiento, especialmente en nuestros países emergentes, por su bajo costo al compararlo con la diálisis ${ }^{7-9}$. Pero, aun así, los programas de trasplante no evolucionan como debe ser, precisamente por el limitado presupuesto en salud y la mucha demanda en salud que hay en nuestra región ${ }^{10,11}$.

En América Central y el Caribe, los programas de trasplante renal, en forma general, están poco desarrollados. La mayor parte de ellos no tienen una estructura nacional de trasplante, o es poco funcional, y no tienen en su mayoría el componente de donante fallecido $0^{10,11}$.

En diversas ocasiones se han llevado a cabo iniciativas tendientes a agrupar a los países de la región y trabajar juntos en el desarrollo de sus respectivos programas de trasplante. Así, en febrero de 1998, se organizó la Sociedad Centroamericana y del Caribe de Trasplante Renal (SOCECATRE), que, por primera vez, reunió en un solo grupo a todos los colegas de la región que trabajaban en trasplante renal, y durante los años que existió fue un vehículo de unión y colaboración entre los países miembros. Más recientemente, a través de la Asociación Centroamericana de Nefrología e Hipertensión (ACECANH), se han reanudado esfuerzos para unir a los países de Centroamérica y el Caribe, y conocer lo que se hace en trasplante renal.

Así, en agosto del 2015, en Heredia, Costa Rica, se realizó la I Reunión de Centroamérica y del Caribe de Trasplante Renal, en la que los representantes de todos los países de la región expusieron la situación en cuanto al trasplante renal en sus respectivos países, y se sentaron las bases para el trabajo conjunto. En agosto del 2017, se realizó la II Reunión de Centroamérica y del Caribe de Trasplante Renal, en San Salvador, con los auspicios y la participación de la Sociedad de Trasplantes de América Latina y el Caribe (STALYC) y la Sociedad Latinoamericana de Nefrología e Hipertensión (SLANH), y en la que se analizó la situación de los trasplantes renales en la región y se llegaron a acuerdos importantes, como la creación del registro de trasplante renal para toda la región. Se escogió una comisión que se encargaría de esta tarea. El objetivo del presente trabajo es exponer los datos de este primer registro regional de trasplante.

\section{Material y método}

La comisión designada para elaborar el registro de trasplante renal de la región, integrada por representantes de todos los países del área, elaboró un borrador de una plantilla, con la cual se recogería la información de la actividad de trasplante en cada país. Esta fue evaluada por las sociedades locales de trasplante o nefrología, además de un representante de la STALYC y la SLANH. Una vez consensuada, se envió 
a cada país para que en un plazo de dos meses la completaran y devolvieran a la comisión coordinadora de la actividad. La plantilla contiene exactamente los datos que se brindan en el presente trabajo. Estos datos, en algunos países, fueron proporcionados por la estructura nacional de trasplantes, y, en los demás, por las sociedades nacionales de trasplante o nefrología. Todos los países enviaron su información. Estos datos fueron presentados y discutidos durante la reunión de San Salvador.

Datos estadísticos: Se hizo uso de estadística descriptiva, con variables categóricas expresadas en sus valores absolutos o porcentaje.

\section{Resultados}

\section{Componentes del sistema de salud}

Excepto Cuba, que tiene un sistema totalmente público, y Costa Rica, que tiene un sistema de seguridad social que cubre a su población, el resto de los países tiene un sistema mixto público/seguridad social.

\section{Tipos de trasplante que se realizan en cada país}

Todos los países hacen trasplante renal. Excepto el Salvador, los demás hacen de córneas. Países como Costa Rica, República Dominicana y Panamá hacen otros tipos de trasplante, como hígado, corazón y médula ósea.

Todos los países hacen trasplantes renales de donante vivo en forma regular, excepto Nicaragua y Honduras, donde se hacen ocasionalmente. Estos dos países y El Salvador no tienen donación de fallecidos.

Todos los países tienen equipo de trasplante renal de carácter público. Nicaragua, República Dominicana y Guatemala tienen, además, equipos privados.

\section{Ley, consejo o comité nacional y estructura nacional de donación y trasplante}

Los países de la región cuentan con ley de trasplantes, excepto Cuba, que tiene un resuelto ministerial. República Dominicana es el país con la ley más antigua.

A pesar de contar con ley de trasplante, no en todos los países tienen un consejo o comité nacional, entidad que vela precisamente porque se cumpla la ley. Guatemala, Honduras y Nicaragua no cuentan con este organismo.

Panamá, República Dominicana y Cuba cuentan con una estructura nacional de donación y trasplante. En
Costa Rica se han dado los primeros pasos para tener la suya. Estos últimos cuatro países cuentan con coordinadores de trasplante al servicio de la estructura nacional (Tabla 1).

\section{Presupuesto del programa de trasplante renal}

La mayor parte de los países cuentan con una asignación del presupuesto de salud del estado para el programa de trasplante renal. Solo Honduras no cuenta con este presupuesto.

\section{Número de hospitales públicos y privados que hacen trasplante}

Los países de la región cuentan con al menos un hospital público donde se hace trasplante renal. En ese sentido destaca Cuba, que tiene nueve centros, y Costa Rica, que tiene cinco.

Excepto Costa Rica y Cuba, los demás países tienen al menos un hospital privado donde se hacen trasplantes renales. El Salvador y República Dominicana, cada uno con cuatro centros, son los países que más tienen (Tabla 2).

\section{Recursos humanos en trasplante renal por país}

Cada país cuenta al menos con 25 nefrólogos. Cuba y República Dominicana destacan con 324 y 156 nefrólogos, respectivamente.

Igualmente, todos los países tienen, al menos, un cirujano de trasplante renal $y$, al menos, un equipo que hace trasplante renal. Algo a destacar es que, excepto Honduras, todos los países cuentan con un nefropatólogo (Tabla 3).

\section{Pago de la inmunosupresión}

El estado paga el $100 \%$ de la inmunosupresión en todos los países, con excepción de República Dominicana, donde paga el $95 \%$ de los costos. La duración de esa cobertura del estado varía en cada país, desde los seis primeros meses postrasplante hasta la cobertura permanente.

En Guatemala cuentan con una entidad privada, una ONG, que cubre los costos de trasplante renal a algunos pacientes seleccionados por sus bajos ingresos (Tabla 4). 
Tabla 1. Estructura nacional de donación y trasplante por país

\begin{tabular}{|l|l|l|l|l|l|}
\hline País & $\begin{array}{l}\text { Tiene estructura nacional } \\
\text { de donación y trasplante }\end{array}$ & $\begin{array}{l}\text { Está dentro o adscrita al } \\
\text { ministerio de salud }\end{array}$ & $\begin{array}{l}\text { Cuenta con } \\
\text { presupuesto propio }\end{array}$ & $\begin{array}{l}\text { Como se elige a su } \\
\text { encargado }\end{array}$ & $\begin{array}{c}\text { Periodo de } \\
\text { duración } \text { (años) }\end{array}$ \\
\hline Guatemala & No & & & \\
\hline Honduras & No & & Sí. El estado & Designación \\
\hline El Salvador & No (en proceso) & Sínisterial & Por concurso \\
\hline Nicaragua & No & Adscrita & No & Designación \\
\hline Costa Rica & Sí & Sí & Sí & ministerial \\
\hline Panamá & Sí & Sí & Sí & Por votación de los \\
\hline Cuba & Sí & & coordinadores de \\
\hline $\begin{array}{l}\text { República } \\
\text { Dominicanante }\end{array}$
\end{tabular}

Tabla 2. Número de hospitales públicos y privados que hacen trasplante renal

\begin{tabular}{|l|c|c|}
\hline País & $\begin{array}{c}N^{0}{ }^{0} \text { de hospitales } \\
\text { públicos que } \\
\text { hacen trasplante. }\end{array}$ & $\begin{array}{c}N^{0}{ }^{0} \text { de hospitales } \\
\text { privados que } \\
\text { hacen trasplante }\end{array}$ \\
\hline Guatemala & 2 & 3 \\
\hline Honduras & 4 & 1 \\
\hline El Salvador & 1 & 4 \\
\hline Nicaragua & 1 & 2 \\
\hline Costa Rica & 5 & --- \\
\hline Panamá & 1 & 2 \\
\hline Cuba & 9 & --- \\
\hline República Dominicana & 1 & 4 \\
\hline
\end{tabular}

Honduras y Panamá son los únicos países que solo usan inmunosupresores innovadores. Los otros países usan innovadores y genéricos.

\section{Inducción en trasplantes renales}

Todos los países utilizan basiliximab como inductor de rutina. La mayor parte usa timoglobulina en casos seleccionados y solo Guatemala usa Atgam, también en casos seleccionados. Estos inductores se usan tanto en casos de donantes vivos como de donantes fallecidos en la mayoría de los países de la región (Tabla 5).

\section{Lista de espera nacional renal}

Solo Costa Rica, Panamá, Cuba y República Dominicana tienen lista de espera nacional para trasplante renal, y es manejada por la estructura nacional de donación y trasplante.

\section{Registro nacional de trasplante renal}

Cuba y Panamá tienen un registro nacional renal bien llevado por la estructura nacional de donación y trasplante. Costa Rica está iniciándolo y República Dominicana lo lleva parcialmente. El resto de los países no tiene este registro.

\section{Total de trasplantes renales de donante vivo hecho por país}

La tabla 6 muestra el total de trasplantes hechos por país desde el año 2012 a agosto del 2016. Se hicieron 11,443 trasplantes. De estos, 432 fueron privados.

\section{Total de trasplantes renales de donante fallecido hechos por país}

Podemos apreciar, en la tabla 7 , el número de trasplantes de donante fallecido hechos por país, en total 3,254 trasplantes. 
Tabla 3. Recursos humanos en trasplante renal por país

\begin{tabular}{|c|c|c|c|c|c|}
\hline País & $\begin{array}{l}\text { N. }{ }^{0} \text { de equipos } \\
\text { de trasplante }\end{array}$ & $\begin{array}{l}\mathrm{N}^{\circ}{ }^{\circ} \text { de } \\
\text { nefrólogos }\end{array}$ & $\begin{array}{l}\text { N. }{ }^{0} \text { de centros que } \\
\text { forman nefrólogos }\end{array}$ & $\begin{array}{l}\text { N. }{ }^{0} \text { de cirujanos de } \\
\text { trasplante renal }\end{array}$ & $\begin{array}{l}\text { N. }{ }^{0} \text { de nefropatólogos y } \\
\text { lugar de formación }\end{array}$ \\
\hline Guatemala & 2 & 55 & 2 & 5 & 1 en México \\
\hline Honduras & 1 & 27 & 0 & 1 & 0 \\
\hline El Salvador & 1 & 38 & 2 & 2 & 3 en el exterior \\
\hline Nicaragua & 3 & 26 & 0 & 4 & 3 en el exterior \\
\hline Costa Rica & 4 & 25 & 4 & 16 & 4 en el exterior \\
\hline Panamá & 1 & 32 & 2 & 6 & 1 en Estados Unidos \\
\hline Cuba & 9 & 324 & 11 & 18 & 5 en el país \\
\hline República Dominicana & 4 & 156 & 3 & 8 & 2 en México y Venezuela \\
\hline
\end{tabular}

Tabla 4. Pago de la inmunosupresión

\begin{tabular}{|l|r|l|l|}
\hline País & El estado & Entidad privada & El paciente \\
\hline Guatemala & Sí & Sí & No \\
\hline Honduras & Sí, $100 \%$ & No & No \\
\hline El Salvador & Sí, $100 \%$ & No & No \\
\hline Nicaragua & Sí & No & Sí, según el caso \\
\hline Costa Rica & Sí, $100 \%$ & No & No \\
\hline Panamá & Sí, $100 \%$ & No & No \\
\hline Cuba & Sí, $100 \%$ & No & No \\
\hline $\begin{array}{l}\text { República } \\
\text { Dominicana }\end{array}$ & Sí, $95 \%$ & $\begin{array}{l}5 \% \text { seguros } \\
\text { privados }\end{array}$ & No \\
\hline
\end{tabular}

\section{Total de trasplantes renales hechos en la región}

Al consolidar los trasplantes renales hechos en el periodo de 2012 a 2017 en la región, tanto de vivos como de fallecidos, tenemos que se han hecho 14,697 trasplantes (Tabla 8).

\section{Total de otros trasplantes no renales en la región}

Adicional a los trasplantes renales, se contabilizaron en la región los trasplantes hepáticos y de corazón hechos por los países que cuentan con estas modalidades. Para el periodo de 2012 a 2016 se hicieron 409 hepáticos y 141 de corazón.

\section{Laboratorios de trasplante}

Los países cuentan al menos con un laboratorio de trasplantes, en esto se excluye a Honduras. Panamá es el único que tiene centralizada esta actividad en un solo laboratorio, que es nacional y sirve a todos los programas de trasplante (Tabla 9).

\section{Estudios inmunológicos realizados en los países de la región}

En la tabla 10 podemos apreciar los estudios inmunológicos que se hacen en cada país y qué países hacen tipificación del antígeno mayor de histocompatibilidad (HLA) por serología, por secuenciación y por DNA. Igualmente, qué países hacen prueba cruzada por citotoxicidad o por citometría de flujo; qué países miden el porcentaje del panel reactivo de antígenos por serología o Luminex, y, además, las novedosas mediciones de anticuerpos anti-HLA por antígeno único pre y postrasplante.

\section{Discusión}

Los países de la región tienen un componente de salud público representado por las entidades estatales del ministerio de salud y la seguridad social. En cada uno varía el porcentaje de población que cubre, con situaciones extremas predominantemente en aquellos donde la menor parte de la población está cubierta por la seguridad social. Panamá es una excepción a esto, ya que la mayor parte de su población está bajo el régimen de seguridad social. Cuba no tiene seguridad 
Tabla 5. Inducción en trasplantes renales

\begin{tabular}{|l|l|l|l|l|}
\hline País & Uso de rutina & Uso ocasional & En receptores de DV & En receptores de DF \\
\hline Guatemala & Seguro social & Salud pública y ONG & Sí & Sí \\
\hline Honduras & Sí & No aplica & Sí & No aplica \\
\hline El Salvador & Trasplantes pediátricos & Sí & Sí & No aplica \\
\hline Nicaragua & Sí & No aplica & Sí & No aplica \\
\hline Costa Rica & Sí & No aplica & Sí & Sí \\
\hline Panamá & Sí & No aplica & Sí & Sí \\
\hline Cuba & Sí $(95 \%)$ & No aplica & Sí & Sí \\
\hline República Dominicana & Sí & No aplica & Sí & Sí \\
\hline
\end{tabular}

DF: donante fallecido; DV: donante vivo; ONG: Organizaciones no gubernamentales.

Fuente: ACECANH. II Encuesta de Centroamérica y Caribe de Trasplante Renal. Agosto 2017.

Tabla 6. Total de trasplantes renales de donante vivo hechos por país

\begin{tabular}{|l|c|c|c|c|c|c|c|}
\hline País & Antes del 2012 & 2012 & 2013 & 2014 & 2015 & 2016 & TOTAL \\
\hline Guatemala & 0 & 0 & 0 & 1,194 (antes de 2015) & 97 & 114 & 1,405 \\
\hline Honduras & 0 & 0 & 0 & 0 & 4 & 9 & 13 \\
\hline El Salvador & 743 & 48 & 48 & 43 & 47 & 63 & 992 \\
\hline Nicaragua & 81 & 12 & 12 & 10 & 10 & 4 & 129 \\
\hline Costa Rica & 1,648 & 89 & 70 & 57 & 49 & 66 & 1,979 \\
\hline Panamá & 201 & 15 & 14 & 10 & 5 & 6 & 251 \\
\hline Cuba & 5,504 & 121 & 18 & 28 & 12 & 25 & 5,708 \\
\hline República Dominicana & 821 & 25 & 27 & 38 & 26 & 29 & 966 \\
\hline TOTAL & & & & & 11,443 \\
\hline
\end{tabular}

Fuente: ACECANH. II Encuesta de Centroamérica y Caribe de Trasplante Renal. Agosto 2017.

social y Costa Rica tiene cubierta a toda su población dentro de la seguridad social.

En cuanto a los tipos de trasplante que se hacen en la región, todos hacen renal y córneas, exceptuando El Salvador que no hace córneas. Algunos países, como Costa Rica, Panamá, República Dominicana y Cuba, han avanzado hacia otros tipos de trasplante, como hepático y corazón. En algunos de estos países también se hace de médula ósea.

Ya entrando en el trasplante renal, todos comenzaron con la modalidad de donante vivo. Honduras, El Salvador y Nicaragua no tienen las facilidades para hacer de donante fallecido.

La región, en forma general, atraviesa por problemas comunes que no permiten desarrollar adecuadamente los trasplantes renales. Primero fue la carencia de una ley marco de la actividad. Ya todos los países tienen una ley de trasplante que no se ha implementado y, por ende, no se cumple en sus partes fundamentales. No hay una asignación presupuestaria para la actividad, y donde la hay es insuficiente. No se cuenta con una estructura nacional de donación y trasplante, salvo algunas excepciones, y, aunque la mayor parte de los países tienen médicos entrenados en coordinación de trasplante, no pueden desarrollar sus funciones por diversas razones de tipo administrativo o de organización.

A todos los niveles, ministerios de salud, Consejo de Ministros de Salud de Centroamérica (COMISCA), sociedades médicas, se ha llevado esta situación pero aún no hay una respuesta positiva y medible. 
Tabla 7. Total de trasplantes renales de donante fallecido por país

\begin{tabular}{|l|c|c|c|c|c|c|c|}
\hline País & Antes del 2012 & 2012 & 2013 & 2014 & 2015 & 2016 & TOTAL \\
\hline Guatemala & 0 & 0 & 0 & 164 (antes de 2015) & 20 & 15 & 199 \\
\hline Honduras & 0 & 0 & 0 & 0 & 0 & 0 & 0 \\
\hline El Salvador & 0 & 0 & 0 & 0 & 0 & 0 & 0 \\
\hline Nicaragua & 0 & 0 & 0 & 0 & 0 & 0 & 0 \\
\hline Costa Rica & 706 & 44 & 45 & 48 & 63 & 41 & 947 \\
\hline Panamá & 285 & 45 & 39 & 36 & 28 & 47 & 480 \\
\hline Cuba & 754 & 119 & 110 & 147 & 173 & 160 & 1463 \\
\hline República Dominicana & 43 & 21 & 23 & 40 & 11 & 27 & 165 \\
\hline TOTAL & & & & & 3,254 \\
\hline
\end{tabular}

Fuente: ACECANH. II Encuesta de Centroamérica y Caribe de Trasplante Renal. Agosto 2017.

Tabla 8. Total de trasplantes renales hechos en la región

\begin{tabular}{|l|c|c|c|}
\hline País & Donante vivo & Donante fallecido & TOTAL \\
\hline Guatemala & 1,405 & 199 & 1,604 \\
\hline Honduras & 13 & 0 & 13 \\
\hline El Salvador & 992 & 0 & 992 \\
\hline Nicaragua & 129 & 0 & 129 \\
\hline Costa Rica & 1,979 & 947 & 2,926 \\
\hline Panamá & 251 & 480 & 731 \\
\hline Cuba & 5,708 & 1,463 & 7,171 \\
\hline $\begin{array}{l}\text { República } \\
\text { Dominicana }\end{array}$ & 966 & 165 & 1,131 \\
\hline TOTAL & 11,443 & 3,254 & 14,697 \\
\hline
\end{tabular}

Fuente: ACECANH. II Encuesta de Centroamérica y Caribe de Trasplante Renal. Agosto 2017.

Cuba y Panamá cuentan con una estructura nacional de donación y trasplante y un programa de donante fallecido bien organizado. Costa Rica da los primeros pasos en ese sentido. Guatemala no cuenta con una organización nacional para desarrollar esta modalidad, y lo que hacen es por iniciativa local de algunos hospitales. República Dominicana, a pesar de contar con una estructura nacional, aún no tiene la donación de fallecidos adecuadamente desarrollada.

Sí se ha avanzado notoriamente en cuanto a la capacitación del recurso humano para trasplantes renales. Todos los países, a excepción de Honduras,
Tabla 9. Laboratorios de trasplante por país

\begin{tabular}{|l|c|}
\hline País & N. ${ }^{0}$ de laboratorios en el país \\
\hline Guatemala & 4 \\
\hline Honduras & 0 \\
\hline El Salvador & 1 \\
\hline Nicaragua & 1 \\
\hline Costa Rica & 4 \\
\hline Panamá & 1 \\
\hline Cuba & 4 \\
\hline República Dominicana & 3 \\
\hline
\end{tabular}

Fuente: ACECANH. II Encuesta de Centroamérica y Caribe de Trasplante Renal. Agosto 2017.

cuentan al menos con un equipo humano para hacer estos trasplantes, lo que incluye un nefropatólogo, o contratan estos servicios fuera del país.

En todos los países, menos Cuba, se hacen trasplantes renales de donante vivo en forma privada esporádicamente, en función de que el receptor tenga las facilidades económicas para sufragarlo.

Con relación a la existencia de una lista de espera, en los países que la tienen, esta es de carácter nacional y la maneja la estructura de donación y trasplante. Cuba y Panamá tienen una lista de espera formal que incluye a todos los pacientes renales que cumplen los requisitos para ello. Costa Rica está en proceso de organización de su lista de espera y en República 
Tabla 10. Estudios Inmunológicos realizados en los países de la región

\begin{tabular}{|c|c|c|c|c|c|c|c|c|}
\hline Tipo de estudio & Guatemala & Honduras & $\begin{array}{c}\text { EI } \\
\text { Salvador }\end{array}$ & Nicaragua & $\begin{array}{l}\text { Costa } \\
\text { Rica }\end{array}$ & Panamá & Cuba & $\begin{array}{l}\text { República } \\
\text { Dominicana }\end{array}$ \\
\hline $\begin{array}{l}\text { Tipificación HLA por } \\
\text { serología }\end{array}$ & $x$ & $\begin{array}{l}\text { No se } \\
\text { hace }\end{array}$ & $x$ & $100 \%$ & $100 \%$ & No se hace & $\begin{array}{l}\text { No se } \\
\text { hace }\end{array}$ & $100 \%$ \\
\hline Tipificación HLA por DNA & $x$ & $\begin{array}{l}\text { No se } \\
\text { hace }\end{array}$ & $\begin{array}{l}\text { No se } \\
\text { hace }\end{array}$ & $\begin{array}{l}\text { No se } \\
\text { hace }\end{array}$ & $x$ & $100 \%$ & $100 \%$ & No se hace \\
\hline $\begin{array}{l}\text { Tipificación HLA por } \\
\text { secuenciación }\end{array}$ & No se hace & $\begin{array}{l}\text { No se } \\
\text { hace }\end{array}$ & $\begin{array}{l}\text { No se } \\
\text { hace }\end{array}$ & $\begin{array}{l}\text { No se } \\
\text { hace }\end{array}$ & $x$ & $100 \%$ & $\begin{array}{l}\text { Solo } \\
\text { médula }\end{array}$ & No se hace \\
\hline Pruebas cruzadas por CDC & $x$ & $\begin{array}{l}\text { No se } \\
\text { hace }\end{array}$ & $100 \%$ & $100 \%$ & $100 \%$ & $\begin{array}{l}\text { Se hace por } \\
\text { fase sólida } \\
\text { DSA } 100 \%\end{array}$ & $30 \%$ & $100 \%$ \\
\hline $\begin{array}{l}\text { Pruebas cruzadas por } \\
\text { citometría de flujo }\end{array}$ & $x$ & $\begin{array}{l}\text { No se } \\
\text { hace }\end{array}$ & $100 \%$ & $\begin{array}{l}\text { No se } \\
\text { hace }\end{array}$ & $x$ & No se hace & $70 \%$ & No se hace \\
\hline $\begin{array}{l}\text { Medición del \% PRA por } \\
\text { serología (CDC) }\end{array}$ & $x$ & $\begin{array}{l}\text { No se } \\
\text { hace }\end{array}$ & $\begin{array}{l}\text { No se } \\
\text { hace }\end{array}$ & $\begin{array}{l}\text { No se } \\
\text { hace }\end{array}$ & $x$ & No se hace & & $95 \%$ \\
\hline $\begin{array}{l}\text { Medición del \% PRA por } \\
\text { Luminex }\end{array}$ & $x$ & $\begin{array}{l}\text { No se } \\
\text { hace }\end{array}$ & $\begin{array}{l}\text { No se } \\
\text { hace }\end{array}$ & $\begin{array}{l}\text { No se } \\
\text { hace }\end{array}$ & $50 \%$ & $100 \%$ & $100 \%$ & $5 \%$ \\
\hline $\begin{array}{l}\text { Medición de anticuerpos } \\
\text { anti-HLA por antígeno } \\
\text { único (single antigen) } \\
\text { "PRETRASPLANTE» }\end{array}$ & No se hace & $\begin{array}{l}\text { No se } \\
\text { hace }\end{array}$ & $\begin{array}{l}\text { No se } \\
\text { hace }\end{array}$ & $\begin{array}{l}\text { No se } \\
\text { hace }\end{array}$ & $30 \%$ & $100 \%$ & $100 \%$ & No se hace \\
\hline $\begin{array}{l}\text { Medición de anticuerpos } \\
\text { anti-HLA por antígeno } \\
\text { único (single antigen) } \\
\text { "POSTRASPLANTE» }\end{array}$ & $\begin{array}{l}\text { Uso postrasplante } \\
\text { sospecha } \\
\text { de rechazo humoral }\end{array}$ & & $20 \%$ & $\begin{array}{l}\text { No se } \\
\text { hace }\end{array}$ & $30 \%$ & $100 \%$ & $100 \%$ & No se hace \\
\hline
\end{tabular}

CDC: prueba de citotoxicidad; DSA: prueba cruzada donante específico; HLA: antígeno mayor de histocompatibilidad; PRA: panel reactivo de antígenos; X: se desconoce el porcentaje.

Fuente: ACECANH. II Encuesta de Centroamérica y Caribe de Trasplante Renal. Agosto 2017.

Dominicana su lista de espera es parcial, algunos hospitales manejan su propia lista de espera.

En cuanto a un registro nacional de trasplante renal, solo Cuba y Panamá cuentan con él, debidamente organizado y funcional. En República Dominicana, este es incompleto y en proceso de organización para que sea nacional. Costa Rica ha dado pasos importantes tendientes a contar con este registro.

En el resto de los países aún se trabaja solicitando la información a los hospitales, usualmente por parte de la sociedad de nefrología local. Uno de los grandes objetivos de la reunión de El Salvador es, a corto plazo, contar con los registros nacionales en los países que no lo tienen, y que esta información fluya hacia la comisión respectiva de la ACECANH, para que se cuente con un registro de la región.

Otro avance, si bien parcial, es que se cuenta en la región con inmunosupresión e inducción para los trasplantes renales pagados por el estado. Pero en buena parte de los países esta cobertura es limitada a un breve espacio de tiempo. Esto es algo que hay que superar, ya que no permite el adecuado desarrollo de este tipo de trasplantes. Solo en Costa Rica, Cuba y Panamá la inmunosupresión es financiada por el estado en forma permanente.

Si bien se presenta la actividad de trasplante renal de la región en el periodo 2012 a 2016, ya que no se cuenta con un registro real en todos los países previo a esa fecha, sí se nos brinda una radiografía de esta actividad y que va creciendo con el paso del tiempo. Esta información recogida, además de permitir conocer que se hace en el área, servirá de base para el registro regional de trasplante renal.

El desarrollo de laboratorios de histocompatibilidad en los países del área es un muy buen ejemplo de cómo la cooperación entre países hermanos puede adelantarlos a todos. Panamá, con un laboratorio de trasplante de punta, ha servido y continúa sirviendo como centro de formación del personal que trabaja en estos laboratorios en sus respectivos países. 


\section{Conclusión}

Se presenta la situación actual de los trasplantes renales en los países de América Central, República Dominicana y Cuba. Sus dificultades, obstáculos y avances, así como los datos generales de los trasplantes renales hechos entre el 2012 y el 2016, información que servirá de base para el desarrollo a breve plazo entre otras cosas del registro de trasplantes de la región.

\section{Conflicto de intereses}

Los autores del presente trabajo declaran no tener ningún conflicto de intereses.

\section{Responsabilidades éticas}

Protección de personas y animales. Los autores declaran que para esta investigación no se han realizado experimentos en seres humanos ni en animales.

Confidencialidad de los datos. Los autores declaran que han seguido los protocolos de su centro de trabajo sobre la publicación de datos de pacientes.

Derecho a la privacidad y consentimiento informado. Los autores declaran que en este artículo no aparecen datos de pacientes.

\section{ANEXO}

Puede consultar el material adicional del anexo en: http://doi.org/10.24875/NEFRO.18000039

\section{Bibliografía}

1. Glassock R, Warnock D, Delanaye P. The global burden of chronic kidney disease: estimates, variability and pitfalls. Nat Rev Nephrol. 2017; 13:104-14.

2. Mills KT, Xu Y, Zhang W, Bundy JD, Chen CS, Kelly TN. A systematic analysis of worldwide population-based data on the global burden of chronic kidney disease in. 2010 Kidney Int. 2015;88:950-57.

3. Jha V, Garcia-Garcia G, Iseki K, Li Z, Naicker S, Plattner B, et al. Chronic kidney disease: global dimension and perspectives. Lancet. 2013; 382:260-72

4. Cusumano AM, González MC. Chronic Kidney Disease in Latin America: Time to Improve Screening and Detection. Clin J Am Soc Nephrol. 2008;3(2):594-600.

5. Czyżewski L, Sańko-Resmer J, Wyzgał J, Kurowski A. Assessment of Health-Related Quality of Life of Patients after Kidney Transplantation in Comparison with Hemodialysis and Peritoneal Dialysis. Ann Transplant. 2014;19:576-85.

6. Purnell TS, Auguste P, Crews DC, Lamprea-Montealegre J, Olufade T, Greer R, et al. Comparison of Life Participation Activities Among Adults Treated by Hemodialysis, Peritoneal Dialysis, and Kidney Transplantation: A Systematic Review. Am J Kidney Dis. 2013;62(5):953-73.

7. Danovitch Gabriel. Handbook of kidney transplantation. Lippincott Williams and Wilkins. 2010.

8. Maganto Pavón E. Historia del Trasplante Renal. En: González Martín M García Buitrón JM. Trasplante Renal. Madrid: Ediciones Aula Médica, 2000.

9. Kahan B, Ponticelli C. Principles and practice of renal transplantation. Editorial Martin Donitz. 2001.

10. Morales-Buenrostro LE. Advances and Challenges in Renal Transplantation in Latin America. Clin Nephrol. 2016 Supplement 1;86 (2016) (13):96-100.

11. Santiago - Del Pin Eduardo; Ruiz J. Octavio. "Los Trasplantes en Latinoamérica", en Trasplante de órganos, II edición 1999. JGH editores S. A 


\title{
Estado de los programas de trasplante renal en América Central y el Caribe
}

\author{
State of the kidney transplant program in Central America and the Caribbean
}

\author{
César Cuero ${ }^{1,2 *}$, Manuel Cerdas ${ }^{1,3}$, José J. Castillos ${ }^{1,4}$, Antonio Enamorado ${ }^{1,5}$, Carlos Henríquez ${ }^{1,6}$, \\ Agualuz Hernández ${ }^{1,7}$, Guillermo Álvarez ${ }^{1,8}$, Francisco González ${ }^{1,9}$ y Luis Morales ${ }^{1,10}$
}

${ }^{1}$ Grupo Coordinador de trasplante Renal de América Central y el Caribe, Asociación Centroamericana y del Caribe de Nefrología (ACECAN); ${ }^{2}$ Organización Panameña de Trasplantes, Panamá; ${ }^{3}$ Hospital México, Caja Costarricense de Seguridad Social, Costa Rica; ${ }^{4}$ Hospital General de La Plaza de la Salud, República Dominicana; ${ }^{5}$ Coordinación Nacional de Trasplantes, Cuba; ${ }^{6}$ Hospital Benjamín Bloom, El Salvador; ${ }^{7} \mathrm{Hospital}$ General de Enfermedades, Instituto Guatemalteco de Seguridad Social, Guatemala; ${ }^{8}$ Centro de Diagnóstico Medicina Avanzada y Telemedicina (CEDIMAT), República Dominicana; ${ }^{9}$ Facultad de Medicina, Universidad de la República, Montevideo, Uruguay; ${ }^{10}$ Instituto Nacional de Ciencias Médicas y Nutrición Manuel Zubirán, Ciudad de México, México

\section{Anexo 1}

II Reunión de Centroamérica y Caribe de Trasplante Renal

Registro Regional de Trasplante Encuesta
Esta encuesta contiene 3 páginas. Por favor, asegúrese de llenarla por completo. Cualquier observación sobre alguna respuesta, por favor, hágala dentro de la casilla correspondiente. Al final hay espacio por si quiere abundar.

1. Información general de la situación de los trasplantes en el país.

1.1. El sistema de salud tiene uno, dos o más componentes. Enumere cuáles

1.2. Tipos de trasplante que se realizan en el país

1.3. ¿Se hace trasplante de donante vivo?

1.4. ¿Se hace trasplante renal de donante fallecido?

1.5. ¿Los trasplantes renales son programados u ocasionales?

1.6. N. ${ }^{\circ}$ de equipos de trasplante renal público

1.7. N. ${ }^{\circ}$ de equipos de trasplante renal privados

1.8. ¿Cuenta el país con una ley de trasplante?

1.9. ¿Tiene el país una estructura nacional de donación y trasplantes?

1.10. ¿Está dentro 0 adscrita al ministerio de salud del país?

1.11. ¿Cuenta esta entidad con presupuesto propio? ¿Quién se lo da?

1.12. ¿Cómo se elige a su encargado y por cuánto tiempo?

\section{Correspondencia:}

*César Cuero

E-mail: ccuero@cwpanama.net licencia CC BY-NC-ND (http://creativecommons.org/licenses/by-nc-nd/4.0/).
Fecha de recepción: 02-04-2018 Fecha de aceptación: 27-06-2020 DOI: 10.24875/NEFRO. 18000039
Disponible en internet: 17-12-2020

Nefro Latinoam. 2020;17:1-3 www.nefrologialatinoamericana.com 
1.13. ¿Tiene el país un comité o consejo nacional de trasplante o similar?

1.14. ¿Existen coordinadores de trasplante en el país?

1.15. ¿Los coordinadores de trasplante están bajo el mando de la estructura nacional de donación y trasplantes? En caso negativo, ¿de quién?

1.16. ¿Cuenta el programa de trasplante renal con presupuesto propio dado por el gobierno?

1.17. ¿Hay aportación presupuestaria privada?

1.18. ¿Existe una única lista de espera nacional renal?

1.19. ¿Quién maneja la lista de espera nacional renal

1.20. ¿Tienen los otros programas de trasplante su propia lista de espera? ¿Quién la maneja?

1.21. ¿Se lleva un registro nacional de trasplante renal? De ser cierto, ¿quién lo maneja?

1.22. ¿Se hacen trasplantes renales privados?

1.23. ¿El seguimiento de los pacientes trasplantados es público?

1.24. ¿El seguimiento es privado? ¿Ambos?

1.25. N. ${ }^{\circ}$ de hospitales públicos que hacen trasplante renal

1.26. $N .^{\circ}$ de hospitales privados que hacen trasplante renal

1.27. N. ${ }^{\circ}$ de equipos de trasplante renal

1.28. N. ${ }^{\circ}$ de nefrólogos con que cuenta el país

1.29. N. ${ }^{\circ}$ de centros que forman nefrólogos

1.30. N. ${ }^{\circ}$ de cirujanos de trasplante renal con que cuenta el país

1.31. N. ${ }^{\circ}$ de nefropatólogos con que cuenta el país ¿Formados en el país o en el exterior?

1.32. Costo de un trasplante renal de donante vivo en dólares

1.33. Costo de un trasplante renal de donante fallecido en dólares

1.34. La inmunosupresión la paga: ¿el estado, una entidad privada, el paciente, una ONG?

1.35. ¿En qué porcentaje la paga cada uno?

\section{Estadísticas}

2.1. Total de trasplantes según modalidad

\begin{tabular}{|l|l|l|l|l|l|c|c|}
\hline Tipo de trasplante & $\begin{array}{l}\text { Total de trasplantes renales hechos } \\
\text { desde el inicio hasta el } 2011\end{array}$ & 2012 & 2013 & 2014 & 2015 & 2016 & Total \\
\hline
\end{tabular}

Renal de donante vivo

Renal de donante fallecido

\subsection{Total de trasplantes públicos y privados}

\begin{tabular}{|l|l|l|l|l|l|l|}
\hline Tipo de trasplante & 2012 & 2013 & 2014 & 2015 & Total \\
\hline
\end{tabular}

Renal público

Renal privado

\subsection{Total de otros trasplantes hasta el 2016}

Tipo de trasplante

Hepático

Corazón 


\section{Inmunosupresión}

3.1. ¿Utiliza su país inducción de rutina u ocasional para receptores de trasplante renal?

3.2. Utilizan esos agentes de inducción en:

¿Receptores de donantes vivos?

¿Receptores de donantes fallecidos?

¿Ambos?

3.3. ¿Qué agentes de inducción usan en su país?

- Basiliximab

- Timoglobulina

- ATGAM

- Alemtuzumab

3.4. Los inmunosupresores que se usan son: ¿Innovadores?

¿Genéricos?

¿Ambos?

\section{Laboratorio de trasplantes}

4.1. ¿Cuántos laboratorios de trasplantes existen en su país?

4.1. Completar la tabla respecto a los estudios inmunológicos realizados de rutina en su país (colocar el $\%$ de pacientes en los que se realiza o puede marcar solo una " $X$ » cuando se desconoce)

\section{Tipo de estudio}

Tipificación HLA por serología

Tipificación HLA por DNA

Tipificación HLA por secuenciación

Pruebas cruzadas por CDC

Pruebas cruzadas por citometría de flujo

Medición de \% PRA por serología (CDC)

Medición de \% PRA por Luminex

Medición de anticuerpos anti-HLA por antígeno único (single antigen) "PRETRASPLANTE»

Medición de anticuerpos anti-HLA por antígeno único (single antigen) "POSTRASPLANTE»

4.1. En caso de no existir laboratorio en su país, ¿dónde envían sus estudios inmunológicos?

CDC: prueba de citotoxicidad; DSA: prueba cruzada donante específico; HLA: antígeno mayor de histocompatibilidad; PRA: panel reactivo de antígenos; $X$ : se desconoce el porcentaje. 